\title{
Single-step fabrication of surface waveguides in fused silica with few-cycle laser pulses
}

\author{
Federico J. Furch ${ }^{1}$, W. Dieter Engel ${ }^{1}$, Tobias Witting ${ }^{1}$, Armando \\ Perez-Leija ${ }^{1}$, Marc J. J. Vrakking ${ }^{1}$, and Alexandre \\ Mermillod-Blondin ${ }^{1 *}$ \\ ${ }^{1}$ Max-Born-Institut für Nichtlineare Optik und \\ Kurzzeitspektroskopie, Max-Born-Straße, D-12489 Berlin, \\ Germany \\ ${ }^{*}$ Corresponding author: mermillod@mbi-berlin.de
}

August 30, 2019

\begin{abstract}
Direct laser writing of surface waveguides with ultrashort pulses is a crucial achievement towards all-laser manufacturing of photonic integrated circuits sensitive to their environment. In this Letter, few-cycle laser pulses (with a sub-10 fs duration) are used to produce subsurface waveguides in a non-doped, non-coated fused silica substrate. The fabrication technique relies on laser-induced microdensification below the threshold for nanopore formation. The optical losses of the fabricated waveguides are governed by the optical properties of the superstrate. We have measured losses ranging from less than $0.1 \mathrm{~dB} / \mathrm{mm}$ (air superstrate) up to $2.8 \mathrm{~dB} / \mathrm{mm}$ when immersion oil is applied on top of the waveguide.
\end{abstract}

Among the several fabrication techniques that may be used to produce waveguides in glass substrates [12, fs-laser direct writing 8 is especially attractive because it does not require a cleanroom environment, is cost-effective, and offers a high throughput [12. Fs-laser writing enables full 3d waveguide fabrication in crystals [7, polymers [2] as well as glasses [10]. Although research efforts have been mostly concentrated on volume microprocessing, the direct fabrication of surface waveguides would greatly extend the domain of applications of laser-written photonic integrated chips. The propagation of an optical field in a surface waveguide implies the presence of an evanescent wave which can be exploited for refractive index sensing [19, 20, plasmonic excitation sensing [29] or Fourier-transform spectrometry 22]. However, using fs-laser direct writing on or near the surface of the host material is challenging. Attempts in non-optimized glasses have resulted in surface swelling [6], cracking [19] and 
ablation [33]. In pure fused silica, the direct fabrication of near-surface waveguides with a good refractive index contrast has even been considered impossible [5]. The main reason is that fs-laser direct writing relies on pulse-to-pulse heat accumulation to induce controlled, localized heating of the substrate. After cooling, high-refractive index regions with light-guiding capabilities appear. In this scheme, the magnitude of the stress load produced upon thermal relaxation is a major limitation. Recently, several routes have been explored to enable surface waveguide photoinscription. One strategy consists in using toughened glasses as a host substrate [19, 20]. Another approach is to enhance the photosensitivity of the glass by doping with silver ions [1]. A third method relies on suppressing the air/dielectric interface by bonding a thin glass on top of the sample [5]. Because the bond is ensured by weak van der Waals attractive forces (only manifesting when the sample and the cover glass are in close contact), such a method might only be applicable to planar substrates. In this Letter, we describe the direct fabrication of surface waveguides in fused silica with the help of few-cycle laser pulses. Our method does not rely on pulse-to-pulse heat accumulation, but instead is based on the type 1 laser-matter interaction regime [25] triggered in a grazing incidence irradiation scheme. Modifications induced in the type 1 regime exhibit a smooth, uniform and positive refractive index change $\Delta n$, in contrast to type 2 modifications which are characterized by an important birefringence due to the presence of periodic nanogratings in the irradiated region. Furthermore, we demonstrate the possibility to control the propagation losses by playing on the refractive index of the superstrate which opens a route for the laser-assisted fabrication of non-hermitian microoptical systems 9.

The experimental setup is depicted in Fig. 1. Few-cycle pulses (sub-10 fs duration, central wavelength at $800 \mathrm{~nm}$ ) from a high repetition rate $(400 \mathrm{kHz})$, non-collinear optical parametric amplifier [11] are focused on the surface of a fused silica sample with a grazing incidence. The samples are parallelepipedic and polished to optical quality on all sides. In order to preserve the temporal structure of the laser pulse in the focal plane, we use a gold-coated reflective objective (numerical aperture 0.5) 26]. Such an irradiation scheme provides two focii $F_{1}$ and $F_{2}$ formed by the upper and lower halves of the laser beam, respectively. The presence of a planar air/glass interface induces wavefront distortions and leads to the formation of an aberrated focus $F_{2}$ [18] which is shifted and spread along the propagation axis. The corresponding laser-induced modifications are shown in Fig. 1(b). An analogous distortion happens in the time domain with consequences on the temporal profile of the irradiation [31. Because the spatio-temporal distortions imparted on the lower half of the beam increase with the amount of propagation in the sample, the irradiation characteristics vary when translating the sample along the propagation axis. In order to limit the irradiation to the part of the beam traveling through air only, the lower half of the laser beam was blocked. Furthermore, the entrance of the beam diameter was slightly reduced with the help of an iris, providing an overall transmission of 0.17 for the focusing unit. The fabrication of a single line-shaped microstructure is then straightforward. It suffices to irradiate the surface of the substrate continuously from edge to edge by translating the sample with the 


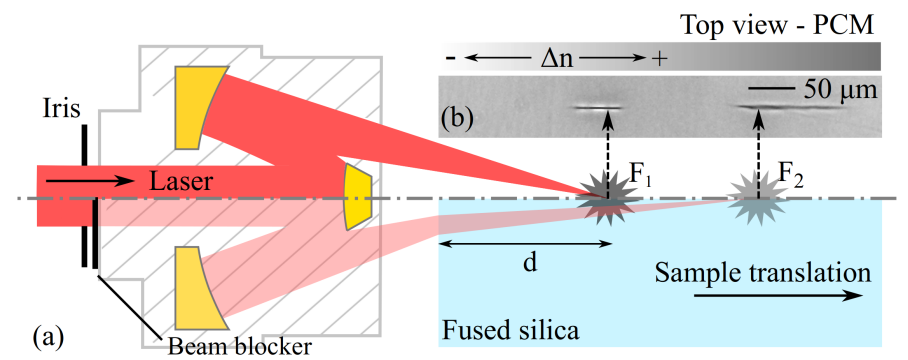

Figure 1: (a): Cross-sectional view of the experimental setup used for laser microprocessing at grazing incidence. The part of the laser beam represented in bright red propagates through air only and forms the focus $F_{1}$. The beam blocker can be put in the beam path to prevent the formation of the aberrated focus $F_{2}$. (b): Phase-contrast microscopy (PCM) picture of the sample's top surface showing the refractive index distribution resulting from $F_{1}$ and $F_{2}$.

help of a stepper motor. In what follows, the laser pulse energy was constant with a value of $E=530 \mathrm{~nJ}$ after the microscope objective and the speed of the stepper motor was $60 \mathrm{um} \mathrm{s}^{-1}$. The laser-induced microstructures had a length of $10 \mathrm{~mm}$ (i.e. the width of the sample). For higher pulse energies, intense laser light scattering occurred and the irradiated region exhibited a mix of negative and positive refractive index changes (not shown). These features are indicative of the type 2 interaction regime characterized by the formation of nanopores [4, 25].

In Fig. 2(a) we show the laser footprint on the exit facet after sample polishing, using optical transmission microscopy (numerical aperture 0.9). The laser-induced microstructure has a width $w \approx 2 \mu \mathrm{m}$ and a height $h \approx 6 \mu \mathrm{m}$. Diagnostics of the top surface with an atomic force microscope (AFM) are presented in Fig. 2(b), and reveal the presence of a shallow surface topography variation $(<-10 \mathrm{~nm}$, about $430 \mathrm{~nm}$ FWHM) on top of the laser-induced microstructure. The negative sign of the topology variation is indicative of a volume reduction (and hence a density increase) in the irradiated area, and is the opposite to what happens when microprocessing is performed in the type 2 regime, with longer ( $35 \mathrm{fs}$ ) and more energetic (about $2 \mu \mathrm{J}$ ) laser pulses where surface swelling as high as $250 \mathrm{~nm}$ was measured [6]. An inversion in the sign of the surface topography is consistent with recent observations reporting a volume reduction of glass cantilevers irradiated in the type 1 regime and a net volume increase of cantilevers irradiated in the type 2 regime [4. We emphasize that the absence of material re-deposition in the AFM pictures hints towards a purely non-ablative process. The phase shift distribution $\Delta \phi$ across the laser-induced microstructure presented in Fig. 2(c) was measured by spatial light interference microscopy [35. As expected from a local density increase, $\Delta \phi$ is positive in the irradiated volume [32]. The corresponding spatial average of the laser-induced 
refractive index change $\overline{\Delta n}=\frac{\lambda_{c} \Delta \phi}{2 \pi h} \approx 0.006$, where $\lambda_{c}=550 \mathrm{~nm}$ is the central wavelength of the illumination source (an halogen light bulb in our case), $h=6 \mu \mathrm{m}$ is defined in Fig. 2(a) and $\Delta \phi=0.43 \mathrm{rad}$ is the phase shift measured at the center of the microstructure. We emphasize that the magnitude of $\overline{\Delta n}$ obtained exceeds the value of $10^{-4}-10^{-3}$ usually measured in the bulk for type 1 modifications [25].
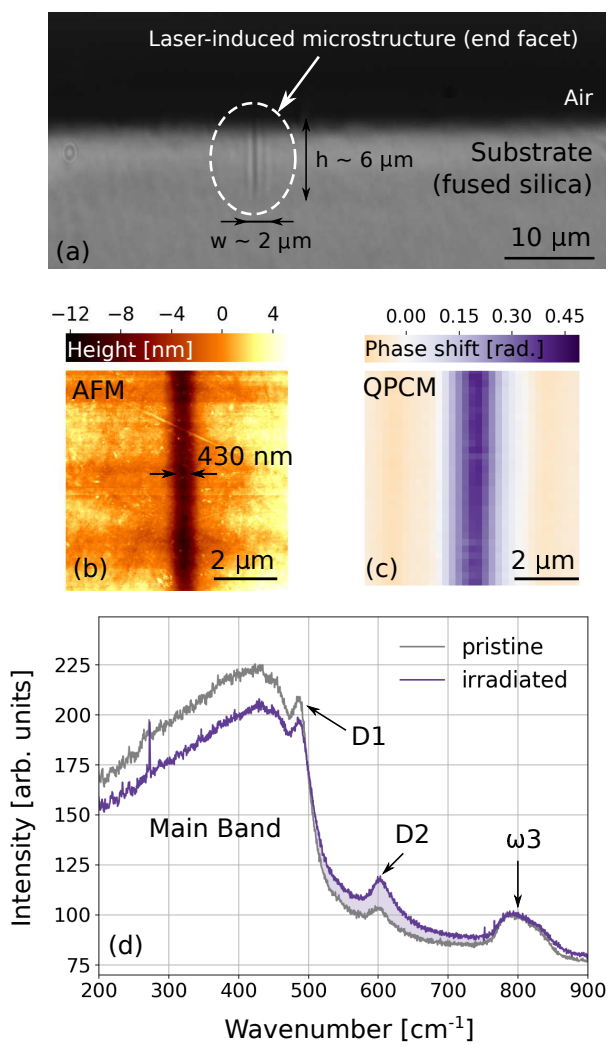

Figure 2: Characterization of the laser-induced optical structures. (a): Side view of the sample acquired with an optical transmission microscope. The sample is illuminated with an halogen lamp. (b): Surface topography (top view) measured with an atomic force microscope (AFM).(c): Phase shift across the microstructure, measured with a spatial light interference microscope (SLIM). (d): Micro-Raman investigations of the irradiated zone (purple line) and of the pristine sample (grey line). The spectra were normalized with respect to the $\omega 3$ band.

To further confirm our conclusion on the laser-induced compaction and refractive index increase, we have examined the signature of the fs laser-induced structural modifications in micro-Raman spectra [25, 30] measured in the pris- 
tine and irradiated material, as shown in Fig. 2(d). The excitation wavelength was $442 \mathrm{~nm}$ and the microscope objective used for excitation and collection of the Raman signal has a numerical aperture of 0.8 which provides a depth resolution of $0.85 \mu \mathrm{m}$. The spectra were normalized with respect to the amplitude of the $\omega 3$ band [28. They reveal an increase of the D2 peak (centered at $600 \mathrm{~cm}^{-1}$ ) in the irradiated volume, confirming a local laser-induced compaction [3, 4]. These micro-Raman measurements were carried out on the top surface of the sample and might not necessarily correspond to the maximum of compaction, presumably located in the center of the laser-induced microstructure (i.e. $\approx 3 \mu \mathrm{m}$ away from the surface).

In order to examine the ability of the microstructures to guide light at optical frequencies, the fundamental mode of a CW He-Ne beam $(\lambda=633 \mathrm{~nm})$ was focused onto the entrance facet of the laser-induced microstructures with a microscope objective (numerical aperture 0.42). A second microscope objective (Olympus MPlan, 100x, numerical aperture 0.9) used in combination with a tube lens (focal length of $200 \mathrm{~mm}$ ) formed a 111-fold magnified image of the end facet on a camera sensor. Figure 3(a) shows the obtained output intensity distribution. It demonstrates that these laser-induced microstructures support optical waveguiding at $633 \mathrm{~nm}$. A modal analysis in the directions parallel $(p-)$ and perpendicular $(s-)$ to the surface of the sample (see Fig. $3(\mathrm{~b})$ ) provides a mode field diameter (MFD, defined as the $1 / e^{2}$ decay of the maximum mode intensity), of 4.3 and $6.0 \mu \mathrm{m}$ in the $p-$ and $s-$ directions, respectively. Out of the center region, the guided mode intensity decays exponentially [16] with decay constants of $\approx 1.1 \mu \mathrm{m}$ and $0.4 \mu \mathrm{m}$ in glass and in air, respectively. We checked that the optical transfer function of the microscope objective did not significantly influence these values by applying a deconvolution algorithm to the curves shown in Fig. 3(b). The point spread function used for the deconvolution was estimated numerically based on the model of Gibson and Lanni [13, 23.

The influence of the input polarization was examined by placing the waveguide between a polarizer and an analyzer. The transmission of the waveguide was measured as a function of the relative angle between the polarizer and the analyzer [see Fig. 3(b)]. The polarization is maintained for input fields with a linear polarization in the s- and p- directions (see thick transparent lines in Fig. 3(b) left). However, an input field with a linear input polarization in another direction becomes elliptically polarized upon propagation in the waveguide, which indicates that s- and p- polarized fields have different propagation constants.

Having confirmed the waveguiding capabilities of the laser-induced microstructures and their polarization-maintaining properties, we now examine the possibility to control the propagation losses by varying the refractive index of the superstrate $n_{s}$. By applying the optimum end-fire coupling method [15], losses $<0.1 \mathrm{~dB} \mathrm{~mm}^{-1}$ were measured for $n_{s}=1.00$ (in air). The losses obtained when applying a drop of immersion oil on top of the waveguide are shown in Fig. 4. The diameter of the oil droplet was controlled by using a graduated microsyringe.

A first order exponential fit of the experimental data indicates that the 
(a)

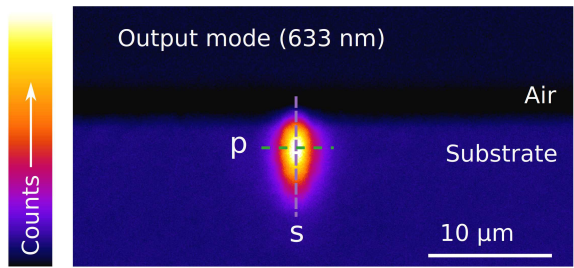

(b)
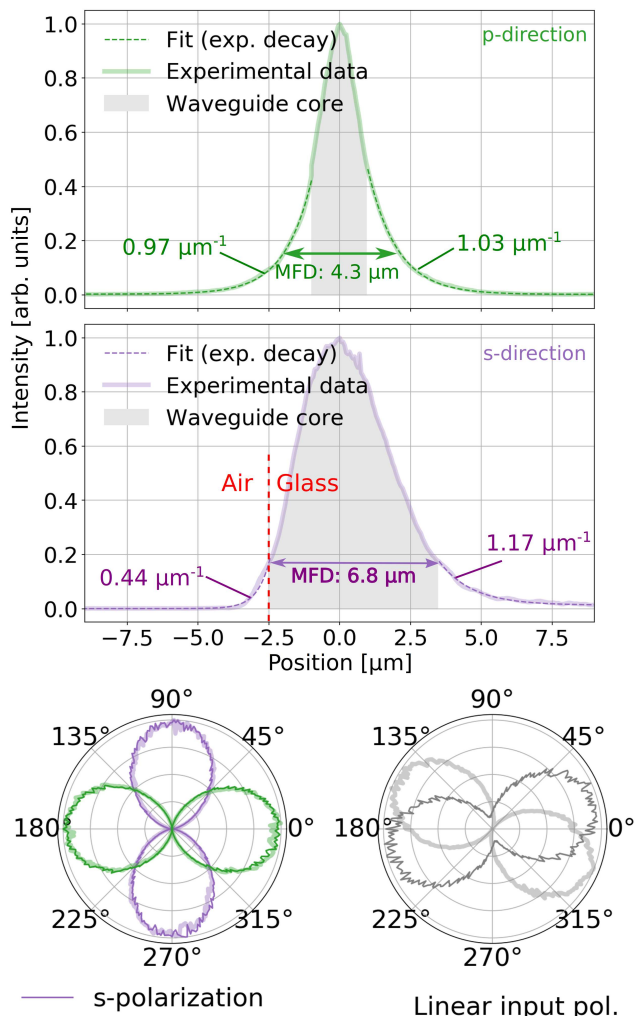

(c)

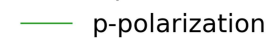
(arbitrary angle)

Figure 3: (a): Near-field intensity profile at the exit of the laser-induced microstructure at a wavelength of $633 \mathrm{~nm}$. (b): Mode-field analysis. The regions in gray correspond to the size of the waveguide core deduced from Fig. 2(a). The dotted lines are first-order exponential fits of the experimental data. MFD: mode field diameter defined as the $1 / e^{2}$ decay of the maximum intensity. (c): Transmitted intensity as a function of the analyzer angle for linear input polarizations in the p- and s- directions (left) and for an arbitrary linear input polarization (right). The dark thin lines represent the polarization of the beam after propagation in the waveguide and the thick, semi transparent lines represent the polarization of the input beam. 


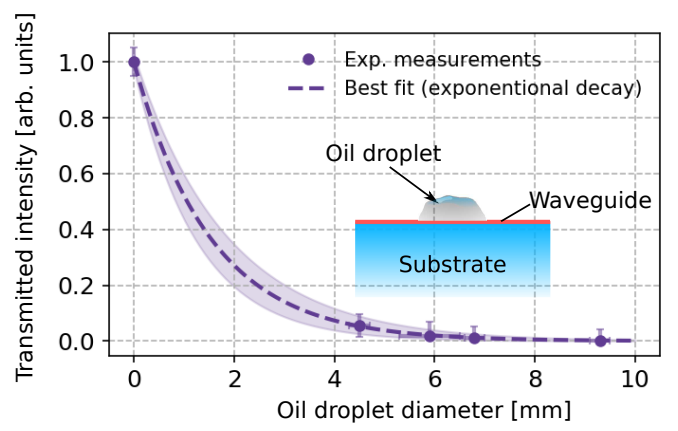

Figure 4: Evolution of the optical transmission at $633 \mathrm{~nm}$ as a droplet of immersion oil $\left(n_{\text {oil }}=1.516\right)$ with a variable diameter is placed on top of the laser-induced surface waveguide. The dotted line represents a fit of the experimental data using a single exponential decay. The presence of oil induces an attenuation of $2.83_{-0.66}^{+0.48} \mathrm{~dB} \mathrm{~mm}^{-1}$.

superstrate-induced leakage is as strong as $2.83_{-0.66}^{+0.48} \mathrm{~dB} \mathrm{~mm}^{-1}$. The uncertainties were obtained from fits of the lower and upper bounds of the experimental values. In the so-called ray-optic approach of guided mode theory [34, the light propagating through a waveguide is described as a sum of oblique rays experiencing total internal reflection at the boundary of the waveguide. Changing $n_{s}$ changes the conditions for total internal reflection. When $n_{s}$ increases, the minimum angle for total internal reflection decreases and the steepest rays escape the waveguide [27, 20].

The large attenuation coefficient deduced from the numerical fit indicates that the optical structures are sensitive to the refractive index of their environment and can thus be employed as refractive index sensors, with significant potential for lab-on-chip applications. These waveguides may for instance constitute the sensitive part of optical biosensors microsystems that are used for label-free bio-sensing [29]. The optical structures presented in this Letter may also be used as efficient photon/plasmon couplers to interface photonic and plasmonic architectures [14, or as the backbone for the fabrication of compact stationary-wave integrated Fourier-transform spectrometers [22]. Furthermore, the ability to control the losses in integrated waveguide configurations opens the door to new perspectives to manufacture non-Hermitian non-resonant photonic systems in connection with exceptional points singularities [24].

In this Letter we have presented a method to inscribe waveguides directly on the surface of a pure fused silica substrate. For the results presented here, few-cycle pulses from a high repetition rate non-collinear optical parametric amplifier have been utilized. The extent to which longer pulses can be utilized will be the subject of future investigations. We note that stretching the pulse by linear dispersion is not a viable option. The highly structured ultra broadband spectrum quickly leads to a multi-pulse structure in the time domain, which 
could potentially change the dynamics of the ionization process. We also note that new trends in non-linear pulse compression have the potential to bring few-cycle pulse capabilities to lasers typically used in laser material processing 21.

The results presented in this Letter represent the first demonstration of waveguides that are directly photoinduced on the surface of fused silica without the need for pre- (e.g. deposition of a photosentive material or applicaton of a cover slip) or post-processing of the target substrate. The optical structures produced correspond to type 1 modifications, support waveguiding at optical frequencies, possesses polarization-maintaining properties and exhibit a core refractive index change of $\Delta n \approx+0.006$ on average. AFM measurements and Micro-Raman investigations indicate that laser-induced microcompaction is at the origin of the observed refractive index change. The waveguides are sensitive to their environment, extending the capability of the direct laser write method to the rapid prototyping of compact optical non-Hermitian microsystems taking advantage of all the well-known benefits (small size and weight, low power consumption, improved reliability and vibration sensitivity [17]) of integrated optical devices.

\section{Funding Information}

Deutsche Forschungsgemeinschaft, Grants ME4427/1-1 and ME4427/1-2.

\section{Acknowledgments}

The authors thank J. Tomm and S. Schwirzke-Schaaf for their assistance with the micro-Raman measurements.

\section{Supplemental Documents}

\section{References}

[1] Alain Abou Khalil, Jean-Philippe Bérubé, Sylvain Danto, Jean-Charles Desmoulin, Thierry Cardinal, Yannick Petit, Réal Vallée, and Lionel Canioni. Direct laser writing of a new type of waveguides in silver containing glasses. Scientific Reports, 7(1):11124, September 2017.

[2] Alexandra Baum, Patricia J. Scully, Maria Basanta, C. L. Paul Thomas, Peter R. Fielden, Nicholas J. Goddard, Walter Perrie, and Paul R. Chalker. Photochemistry of refractive index structures in poly(methyl methacrylate) by femtosecond laser irradiation. Opt. Lett., 32(2):190-192, Jan 2007.

[3] Y. Bellouard, E. Barthel, A. A. Said, M. Dugan, and P. Bado. Scanning thermal microscopy and raman analysis of bulk fused silica exposed to low- 
energy femtosecond laser pulses. Opt. Express, 16(24):19520-19534, Nov 2008.

[4] Yves Bellouard, Audrey Champion, Benjamin McMillen, Sebabrata Mukherjee, Robert R. Thomson, Charles Pépin, Philippe Gillet, and Ya Cheng. Stress-state manipulation in fused silica via femtosecond laser irradiation. Optica, 3(12):1285-1293, Dec 2016.

[5] Jean-Philippe Bérubé and Réal Vallée. Femtosecond laser direct inscription of surface skimming waveguides in bulk glass. Opt. Lett., 41(13):3074-3077, Jul 2016.

[6] V. R. Bhardwaj, P. B. Corkum, D. M. Rayner, C. Hnatovsky, E. Simova, and R. S. Taylor. Stress in femtosecond-laser-written waveguides in fused silica. Opt. Lett., 29(12):1312-1314, Jun 2004.

[7] Feng Chen and J. R. Vázquez de Aldana. Optical waveguides in crystalline dielectric materials produced by femtosecond-laser micromachining. Laser ES Photonics Reviews, 8(2):251-275, 2014.

[8] K. M. Davis, K. Miura, N. Sugimoto, and K. Hirao. Writing waveguides in glass with a femtosecond laser. Opt. Lett., 21(21):1729-1731, 1996.

[9] Ramy El-Ganainy, Mercedeh Khajavikhan, Demetrios N. Christodoulides, and Sahin K. Ozdemir. The dawn of non-hermitian optics. Communications Physics, 2(1):37, March 2019.

[10] C. Florea and K. A. Winick. Fabrication and characterization of photonic devices directly written in glass using femtosecond laser pulses. Journal of Lightwave Technology, 21(1):246-253, Jan 2003.

[11] Federico J. Furch, Achut Giree, Felipe Morales, Alexandria Anderson, Yicheng Wang, Claus Peter Schulz, and Marc J. J. Vrakking. Close to transform-limited, few-cycle $12 \mu \mathrm{j}$ pulses at $400 \mathrm{khz}$ for applications in ultrafast spectroscopy. Opt. Express, 24(17):19293-19310, Aug 2016.

[12] Andrea Chiappini Giancarlo C. Righini. Glass optical waveguides: a review of fabrication techniques. Optical Engineering, 53:53 - 53 - 15, 2014.

[13] Sarah Frisken Gibson and Frederick Lanni. Diffraction by a circular aperture as a model for three-dimensional optical microscopy. J. Opt. Soc. Am. A, 6(9):1357-1367, Sep 1989.

[14] Xin Guo, Min Qiu, Jiming Bao, Benjamin J. Wiley, Qing Yang, Xining Zhang, Yaoguang Ma, Huakang Yu, and Limin Tong. Direct coupling of plasmonic and photonic nanowires for hybrid nanophotonic components and circuits. Nano Letters, 9(12):4515-4519, 2009. PMID: 19995088.

[15] M. Haruna, Y. Segawa, and H. Nishihara. Nondestructive and simple method of optical-waveguide loss measurement with optimisation of endfire coupling. Electronics Letters, 28(17):1612-1613, 1992. 
[16] Jonathan $\mathrm{Hu}$ and Curtis R. Menyuk. Understanding leaky modes: slab waveguide revisited. Adv. Opt. Photon., 1(1):58-106, Jan 2009.

[17] Robert G. Hunsperger. Integrated Optics Theory and Technology 6th Edition. Springer, 2009.

[18] N. Huot, R. Stoian, A. Mermillod-Blondin, C. Mauclair, and E. Audouard. Analysis of the effects of spherical aberration on ultrafast laser-induced refractive index variation in glass. Opt. Express, 15(19):12395-12408, SEP 172007.

[19] Jerome Lapointe, Mathieu Gagné, Ming-Jun Li, and Raman Kashyap. Making smart phones smarter with photonics. Opt. Express, 22(13):1547315483, Jun 2014.

[20] Jerome Lapointe, Francois Parent, Elton Soares de Lima Filho, Sébastien Loranger, and Raman Kashyap. Toward the integration of optical sensors in smartphone screens using femtosecond laser writing. Opt. Lett., 40(23):5654-5657, Dec 2015.

[21] L. Lavenu, M. Natile, F. Guichard, Y. Zaouter, X. Delen, M. Hanna, E. Mottay, and P. Georges. Nonlinear pulse compression based on a gasfilled multipass cell. Opt. Lett., 43(10):2252-2255, May 2018.

[22] Etienne Le Coarer, Sylvain Blaize, Pierre Benech, Ilan Stefanon, Alain Morand, Gilles Lerondel, Gregory Leblond, Pierre Kern, Jean Marc Fedeli, and Pascal Royer. Wavelength-scale stationary-wave integrated fouriertransform spectrometry. Nat Photon, 1(8):473-478, August 2007.

[23] Jizhou Li, Feng Xue, and Thierry Blu. Fast and accurate three-dimensional point spread function computation for fluorescence microscopy. J. Opt. Soc. Am. A, 34(6):1029-1034, Jun 2017.

[24] Mohammad-Ali Miri and Andrea Alù. Exceptional points in optics and photonics. Science, 363(6422), 2019.

[25] K. Mishchik, C. D'Amico, P. K. Velpula, C. Mauclair, A. Boukenter, Y. Ouerdane, and R. Stoian. Ultrafast laser induced electronic and structural modifications in bulk fused silica. Journal of Applied Physics, 114(13):133502, 2013.

[26] B. Piglosiewicz, D. Sadiq, M. Mascheck, S. Schmidt, M. Silies, P. Vasa, and C. Lienau. Ultrasmall bullets of light-focusing few-cycle light pulses to the diffraction limit. Opt. Express, 19(15):14451-14463, Jul 2011.

[27] F. Rehouma, D. Persegol, and A. Kevorkian. Optical waveguides for evanescent field sensing. Applied Physics Letters, 65(12):1477-1479, 1994. 
[28] R. Saavedra, M. León, P. Martin, D. Jiménez-Rey, R. Vila, S. Girard, A. Boukenter, and Y. Ouerdane. Raman measurements in silica glasses irradiated with energetic ions. AIP Conference Proceedings, 1624(1):118$124,2014$.

[29] B Sepúlveda, J Sánchez del Río, M Moreno, F J Blanco, K Mayora, C Domínguez, and L M Lechuga. Optical biosensor microsystems based on the integration of highly sensitive machzehnder interferometer devices. Journal of Optics A: Pure and Applied Optics, 8(7):S561, 2006.

[30] N. S. Shcheblanov, M. E. Povarnitsyn, K. N. Mishchik, and A. Tanguy. Raman spectroscopy of femtosecond multipulse irradiation of vitreous silica: Experiment and simulation. Phys. Rev. B, 97:054106, Feb 2018.

[31] Bangshan Sun, Patrick S. Salter, and Martin J. Booth. Effects of sample dispersion on ultrafast laser focusing. J. Opt. Soc. Am. B, 32(7):1272-1280, Jul 2015.

[32] C.Z. Tan, J. Arndt, and H.S. Xie. Optical properties of densified silica glasses. Physica B: Condensed Matter, 252(12):28 - 33, 1998.

[33] Gustavo A. Torchia, Pablo F. Meilán, Airan Rodenas, Daniel Jaque, Cruz Mendez, and Luis Roso. Femtosecond laser written surface waveguides fabricated in nd:yag ceramics. Opt. Express, 15(20):13266-13271, Oct 2007.

[34] R. Ulrich and R. J. Martin. Geometrical optics in thin film light guides. Appl. Opt., 10(9):2077-2085, Sep 1971.

[35] Zhuo Wang, Larry Millet, Mustafa Mir, Huafeng Ding, Sakulsuk Unarunotai, John Rogers, Martha U. Gillette, and Gabriel Popescu. Spatial light interference microscopy (slim). Opt. Express, 19(2):1016-1026, Jan 2011. 